\title{
Real-Time 3D Tracking and Reconstruction on Mobile Phones
}

\author{
Victor Adrian Prisacariu, Member, IEEE, Olaf Kähler, Member, IEEE, \\ David W. Murray, Member, IEEE, and Ian D. Reid, Member, IEEE
}

\begin{abstract}
We present a novel framework for jointly tracking a camera in 3D and reconstructing the 3D model of an observed object. Due to the region based approach, our formulation can handle untextured objects, partial occlusions, motion blur, dynamic backgrounds and imperfect lighting. Our formulation also allows for a very efficient implementation which achieves real-time performance on a mobile phone, by running the pose estimation and the shape optimisation in parallel. We use a level set based pose estimation but completely avoid the, typically required, explicit computation of a global distance. This leads to tracking rates of more than $100 \mathrm{~Hz}$ on a desktop PC and $30 \mathrm{~Hz}$ on a mobile phone. Further, we incorporate additional orientation information from the phone's inertial sensor which helps us resolve the tracking ambiguities inherent to region based formulations. The reconstruction step first probabilistically integrates $2 \mathrm{D}$ image statistics from selected keyframes into a $3 \mathrm{D}$ volume, and then imposes coherency and compactness using a total variational regularisation term. The global optimum of the overall energy function is found using a continuous max-flow algorithm and we show that, similar to tracking, the integration of per voxel posteriors instead of likelihoods improves the precision and accuracy of the reconstruction.
\end{abstract}

Index Terms-3d tracking, 3d reconstruction, augmented reality, mobile phone

\section{INTRODUCTION}

The 3D modelling of objects from 2D images is a central problem in computer vision with far reaching applications in computer graphics. While much work has been dedicated to this problem in recent years, typical solutions often still require powerful hardware [9], specialized and calibrated camera setups with controlled lighting [8], or very accurate 2D object segmentations [1]. These constraints restrict the applicability of 3D modelling from images to a small group of expert users. A less constrained solution could make the technique available to a much wider audience, as happened, for example, in the cases of panorama stitching, nowadays a standard feature of consumer grade digital cameras, and 3D articulated pose recovery, cheaply available from the Microsoft Kinect.

In this paper we aim to provide a reconstruction system that (i) can work in a real world environment under realistic conditions and (ii) has a low enough computational cost to allow it to run in real time on a mobile phone, without any additional specialised hardware. As all processing is done on a wire-less device, the user can move freely around the object, while receiving immediate feedback on the reconstructed $3 \mathrm{D}$ object shape in the phone's display. However, this means we not only have to recover the 3D shape of the object, but also the 3D trajectory of the camera along with its orientation. We opt to run the reconstruction

- Victor Adrian Prisacariu, Olaf Kähler and David W. Murray are with the Department of Engineering Science, University of Oxford,

E-mail: [victor, olaf,dwm@robots.ox.ac.uk]

- Ian D. Reid is with the University of Adelaide.

E-mail: [ian.reid@adelaide.edu.au] and tracking tasks simultaneously and in parallel.

Tracking the camera pose is region based and, on an abstract level, its goal is to find a $3 \mathrm{D}$ pose relative to the object that provides maximum separation of foreground and background areas, which are determined using given image statistics. Such an approach provides robustness against a wide range of image artefacts, including partial object occlusions and motion blur. From a practical viewpoint we use iterative nonlinear optimization methods. The tracker repeatedly renders the $3 \mathrm{D}$ model, computes a level set embedding function (i.e. distance transform) of the rendering and takes a step to increase the overlap. Related tracking approaches [16], [14], [17] therefore typically have very high computation costs and require a powerful GPU to run in real time. Instead, we propose an alternative formulation that avoids the computation of the global distance transform and its derivatives, and gains further efficiency from a hierarchical rendering pipeline. Our tracker also makes use of the additional orientation information that is readily available from the inertial sensor on a typical mobile phone. Overall the implementation achieves real time performance (>30 fps) on a mobile phone or much higher framerates ( $>100 \mathrm{fps}$ ) on a standard desktop PC, without requiring a GPU.

The reconstruction of the 3D object is internally split into two phases. For a selected but fairly dense number of keyframes the extracted 2D foreground and background probability maps are reprojected and accumulated in their respective $3 \mathrm{D}$ probability volumes. These volumes represent the probabilities of $3 \mathrm{D}$ points being situated inside or outside the object. In contrast to previous works we therefore do not require a discrete object segmentation at every frame, and can also capture and deal with uncertain 
image segmentations gracefully. Furthermore, we advocate the use of per-voxel posteriors instead of likelihoods, which further increases the accuracy and robustness to imperfect image statistics.

In the second phase of reconstruction we impose shape coherency and compactness for the object. We do this using a globally optimal total variational formulation and find the solution using continuous max-flow. Due to the comparatively high computational complexity of this step we apply it only every couple of keyframes. We show that continuously performing this step is not strictly required to achieve good quality $3 \mathrm{D}$ models.

An early version of this work was presented in the conference paper [13]. Here we dramatically improve the performance of our method with regard to the reconstruction of objects with thin parts by (i) increasing the speed of the frame registration and (ii) using a sliding average to compute voxel likelihoods. This leads to more accurate results and faster convergence. Additionally, we provide more detailed insights into the mathematical formulation, the technical implementation and experimental performance.

We relate our ideas to the current state-of-the-art in tracking and reconstruction in Section 2. Section 3 provides a more detailed overview of our method, driven by a graphical model, and the notation used throughout the rest of the work. The two major components, tracking and reconstruction, are then presented in Sections 4 and 5 . Crucial implementation details for achieving good performance follow in Section 6. An experimental evaluation of the method is performed in Section 7 and we summarise our conclusions in Section 8.

\section{Related Works}

The pose recovery part of our work is related to regionbased 3D tracking, as proposed initially by [17]. In that work, the Chan-Vese level set energy function [22] is minimised using a two step process, first in an unconstrained manner and second with respect to the $6 \mathrm{DoF}$ pose of the known 3D shape. A more recent update to this work replaces the two phase approach with a single-step approximate evolution to get only the pose [19]. Our work is more closely following a variational formulation of the objective from [17], by minimising the pixel-wise posteriors level set energy function of [2]. However, in contrast to the $2 \mathrm{D}$ object tracking done in [2] we directly estimate a 3D pose instead. This idea was first proposed in [14], where a 3D mesh is used to represent the 3D shape. An improved version of this formulation is presented in [16], where the triangle mesh is replaced by a volumetric 3D signed distance transform. In the present work we opted for a similar volumetric representation, which is well suited for the reconstruction step later on, but we otherwise follow a mathematical formulation very similar to [14]. Our main novelty is to present a more efficient method of computing the gradient needed during optimization of the tracking error function. While the previous works of [14] and [16] require powerful GPUs to achieve framerates of at most
$25 \mathrm{fps}$ on desktop PCs, our approximation allows us to get roughly the same speed on a much less powerful mobile phone processor or considerably higher framerates on a desktop PC without a GPU.

Similar to a range of previous works [24], [3], [15] we augment the visual pose tracker with additional information from an inertial sensor. We use only a lightweight fusion mechanism, but the inertial sensor still provides valuable information about the camera rotation, sufficient to resolve the ambiguities in visual tracking [15].

In the object reconstruction part of our framework we make use of the wide range of prior work on recovery of the visual hull from silhouettes, for example [23], [20], [5], [4], [8]. One of the early approaches proposed in [23] locally minimises the reprojection error between 3D surface and observed image intensities by forward projection, making it slow and subject to local minima. More recent methods instead use the reverse strategy of backprojecting 2D image information into 3D volumetric representations. In [20] binary segmentations are extracted from the images and backprojected, whereas non-discrete image statistics are used in [5], [4] and [8]. All of these methods use globally convergent optimization approaches to segment the foreground object out of the 3D volume, graph-cuts in [20], [5], [4] and total variational primal-dual optimisation in [8]. While the reconstruction step in our work is similar to the above in that we backproject image statistics into a 3D volume and then use globally convergent optimization methods to find the 3D object surface, there are some important differences. First, we propose to use voxel posteriors instead of likelihoods and show that this improves the performance and robustness of the method. Second, we use continuous max-flow optimization [25], which is both much faster than the discrete graph cuts of [5], [4], [20] and shows better convergence than the primaldual total variational optimisation from [8]. Finally, unlike all of the aforementioned works, our system estimates the camera poses online using the partially reconstructed object model and we need neither carefully calibrated camera setups, nor controlled lighting or static background environments.

The problem of simultaneously tracking and densely reconstructing an object has also received prior attention. Two recent representative methods are [11] and [1]. In both of them a static background is assumed and feature tracking is used to localize the camera. In [11] a sparse cloud of $3 \mathrm{D}$ points is reconstructed from detected 2D feature points and a convex 3D shape is then extracted using Delaunay tetrahedralisation. Similarly, the camera pose in [1] is estimated from a sparse 3D map using the PTAM system [7], but then the object is segmented in each frame using graph cuts and the segmentations are merged into a 3D volume using an ad-hoc voting based fusion method. As in the aforementioned [20], this requires an explicit and discrete segmentation into foreground and background for each input image. In contrast to these methods, our approach does not make use of a static map of the background and can hence handle partially dynamic 


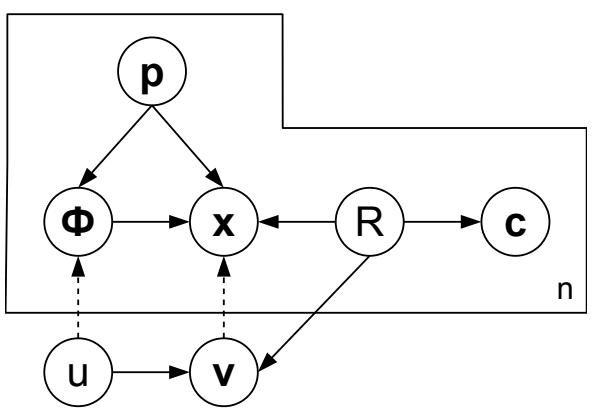

Fig. 1. Graphical model for our method.

scenes, and it does not require feature points, making it more robust to motion blur and partial occlusions.

Another closely related category of research covers systems for simultaneous localisation and mapping (SLAM) with popular examples given in PTAM [7], DTAM [9] and very recently a system presented in [21]. Such systems track and reconstruct the whole scene observed by the camera, producing sparse [7] or dense [9], [21] 3D world maps. Our approach in contrast only creates a model of the actual object that we intend to reconstruct and completely ignores the background. Of course in some cases it will be possible to create a full dense 3D scene model first and then segment the object in the 3D data afterwards. The reconstruction and particularly the tracking might even benefit from incorporating information and additional landmarks from the background. If the fundamental assumption of a static scene background is violated however, the performance of such systems can be expected to break down. This is commonly the case, for example when reconstructing a statue with people walking in the background. Our proposed system completely ignores the scene background and dynamic movements, light changes, and other unmodelled effects in the background and furthermore it does not require strongly textured foreground objects.

\section{Graphical Model}

Figure 1 shows the graphical model describing our method. An overview of the practical implementation is shown and discussed in Section 6. The 3D shape we track and reconstruct is denoted with the random variable $u$. We use a volumetric shape representation, which makes $u$ a 3D volume probability, 0 identifying voxels certainly outside the shape and 1 inside. The maximum likelihood estimate of the outline of the shape is the 0.5 level set of $u$. We denote by $\mathbf{v}$ a distribution over voxels in this volume.

We assume a set of $n$ views. For each of these views, we denote the distribution over 3D poses of the 3D object with p. We use a standard six degree of freedom representation for pose (three for translation and three for Rodrigues parametrised rotation). The contour of the projection of $u$ under the pose $\mathbf{p}$ is embedded inside a $2 \mathrm{D}$ signed distance transform (SDF), which we denote by $\Phi$. Similarly, a voxel location $\mathbf{v}$ under the pose $\mathbf{p}$ projects to a pixel location $\mathbf{x}$. In Figure 1 we denote these deterministic relationships with dotted lines. Note that in this work we consider the $3 \mathrm{D}$ poses to be independently distributed. This could be changed, allowing for a motion model to be added.

Each pixel location $\mathbf{x}$ has a corresponding colour $\mathbf{c}$. As with other region-based methods, we assume a known pair of per-view foreground and background colour models, which we denote by $P(\mathbf{c} \mid R)$ with $R \in\left\{R_{f}, R_{b}\right\}$. Here these are $32 \times 32 \times 32$ bin RGB histograms. $R \in\left\{R_{f}, R_{b}\right\}$ are indicator variables for the foreground and background regions, respectively.

Joint inference on the full graphical model is not practicable, especially on a mobile device. As other works have done before us, we therefore chose to split the inference into a tracking stage, i.e. an estimation of the pose $\mathbf{p}$, and a reconstruction stage, i.e. an estimation of the shape $u$. In the interest of brevity we use $u, \mathbf{v}$ and $\mathbf{p}$ to denote both estimate and respective probability distribution for the remainder of the paper.

\section{Pose Optimisation}

The projection of a known 3D shape $u$ given a pose $\mathbf{p}$, separates any image into a foreground and a background region. Assuming known colour statistics for these regions, the pose optimisation aims to maximise the discrimination between foreground and background with respect to the pose $\mathbf{p}$. The theoretical foundations of this approach have been introduced in [14], and we summarise them in the following.

Treating $u$ and $\mathbf{v}$ as known in the graphical model, the joint probability for a single view becomes similar to the one presented by Bibby and Reid in [2] for the case of 2D tracking and segmentation. This is written as:

$$
P(\mathbf{x}, \mathbf{c}, \mathbf{p}, \Phi, R)=P(\mathbf{x} \mid \mathbf{p}, \Phi, R) P(\mathbf{c} \mid R) P(R) P(\Phi \mid \mathbf{p}) P(\mathbf{p})
$$

In the following we omit $P(\Phi)$ and $P(\mathbf{p})$ as we consider all SDFs and poses equally likely and we omit $\mathbf{p}$ for brevity, as it does not influence the final energy function formulation.

Marginalising wrt. the colour models we obtain:

$$
P\left(\Phi \mid \Omega_{2}\right)=\prod_{\mathbf{x}_{i} \in \Omega_{2}}\left\{\sum_{R} P\left(\mathbf{x}_{i} \mid \Phi, R\right) P(R \mid \mathbf{c})\right\}
$$

with $\Omega_{2}$ being the $2 \mathrm{D}$ image domain and

$$
P\left(\mathbf{x}_{i} \mid \Phi, R_{f}\right)=\frac{H_{e}\left(\Phi\left(\mathbf{x}_{i}\right)\right)}{\eta_{f}} \quad P\left(\mathbf{x}_{i} \mid \Phi, R_{b}\right)=\frac{1-H_{e}\left(\Phi\left(\mathbf{x}_{i}\right)\right)}{\eta_{b}}
$$

where $H_{e}$ denotes the smoothed Heaviside function (commonly used in level set based tracking and segmentation) and $\eta_{f}$ and $\eta_{b}$ are the number of foreground and background pixels, respectively.

The colour posteriors are written as follows:

$$
P\left(R_{j} \mid \mathbf{c}\right)=\frac{P\left(\mathbf{c} \mid R_{j}\right) P\left(R_{j}\right)}{\sum_{i \in f, b} P\left(\mathbf{c} \mid R_{i}\right) P\left(R_{i}\right)} \quad P\left(R_{j}\right)=\frac{\eta_{j}}{\eta}
$$

where $\eta=\eta_{f}+\eta_{b}$ is the total number of pixels in $\Omega_{2}$. This choice of posteriors has been shown in [2] and [14] to 
produce a better separation between foreground and background over the standard approach of using likelihoods, and in turn this leads to more accurate 3D tracking.

Switching to $\log$ probabilities, we write:

$$
\begin{aligned}
E & =\log \left(P\left(\Phi \mid \Omega_{2}\right)\right)= \\
& =\sum_{\mathbf{x}_{i} \in \Omega_{2}} \log \left(H_{e}(\Phi) P_{f}+\left(1-H_{e}(\Phi)\right) P_{b}\right)
\end{aligned}
$$

where:

$$
\begin{aligned}
P_{f} & =\frac{P\left(\mathbf{c} \mid R_{f}\right)}{\eta_{f} P\left(\mathbf{c} \mid R_{f}\right)+\eta_{b} P\left(\mathbf{c} \mid R_{b}\right)} \\
P_{b} & =\frac{P\left(\mathbf{c} \mid R_{b}\right)}{\eta_{f} P\left(\mathbf{c} \mid R_{f}\right)+\eta_{b} P\left(\mathbf{c} \mid R_{b}\right)}
\end{aligned}
$$

This energy function captures the separation between foreground and background with respect to the $2 \mathrm{D}$ shape embedded in $\Phi$. In our case this shape is generated as the projection of the 3D shape $u$ using the pose $\mathbf{p}$. This casts the problem of maximising separation of foreground and background as one of optimising $E$ with respect to $\mathbf{p}$ using standard gradient-based methods. This requires evaluating the following derivative:

$$
\frac{\partial E}{\partial \mathbf{p}}=\sum_{\mathbf{x} \in \Omega_{2}} \frac{\delta_{e}(\Phi)\left(P_{b}-P_{f}\right)}{H_{e}(\Phi) P_{f}+\left(1-H_{e}(\Phi)\right) P_{b}}\left(\frac{\partial \Phi}{\partial x} \frac{\partial x}{\partial \mathbf{p}}+\frac{\partial \Phi}{\partial y} \frac{\partial y}{\partial \mathbf{p}}\right)
$$

with $\delta_{e}$ the derivative of the smoothed Heaviside function and $x$ and $y$ the 2D coordinates of points situated on the contour of the projection of the 3D shape. The remaining derivatives $\partial \Phi / \partial x$ and $\partial \Phi / \partial y$ are computed numerically and $\partial x / \partial \mathbf{p}$ and $\partial y / \partial \mathbf{p}$ follow trivially as detailed in [14].

The framework presented above has been shown to produce state of the art results in region based 3D tracking [14]. This however comes at the expense of high computational cost, as the projection (i.e. rendering) of the 3D shape and its distance transform $\Phi$ have to be computed once per iteration. This means that a real time implementation is only possible using GPU processing. Even so, speeds higher than 20-25 fps are not easily achieved.

In the remaining part of this section we address the three main speed bottlenecks of this approach: (i) the rendering of the 3D shape, (ii) the computation of the SDF and its derivatives and (iii) the optimisation method. We also discuss the issue of silhouette ambiguity, which concerns tracking reliability instead of speed, but is especially important when doing 3D reconstruction.

Hierarchical Binary Rendering. We use a volumetric representation for the shape $u$. The established method for rendering a $3 \mathrm{D}$ shape represented in such a way is to use a raycasting algorithm [9]. Unfortunately this operation is prohibitively slow without GPU hardware, especially on a mobile phone. Our tracker however only needs a binary rendering with depth values only for the pixels located on the edge of that rendering. With this in mind, we chose perform the raycasting operation in a hierarchical manner. We initially raycast a very low resolution image $(40 \times 30$ pixels $)$. We then resize this image by a factor of two, raycast the pixels around the edge and interpolate

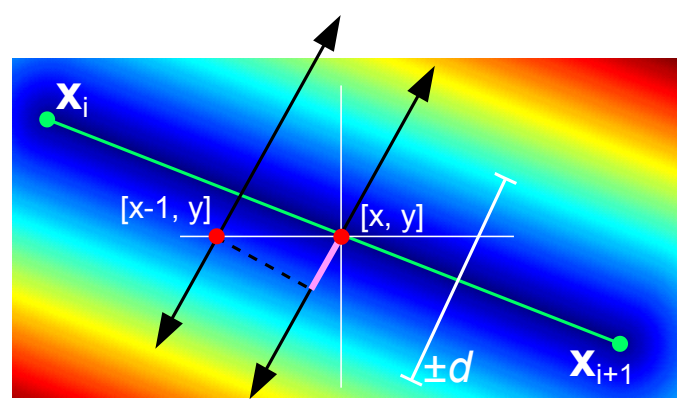

Fig. 2. Geometric explanation for the computation of the derivative of the distance transform.

the others. The process is repeated multiple times until the desired resolution is reached. On a $640 \times 480$ image, this process results in a speedup in excess of $10 \times$ over a standard CPU-based raycast and has the added benefit of producing a resolution hierarchy, that can be used in tracking as shown further down.

Distance Transform and Derivatives. Our pose optimisation requires several computations of a 2D SDF for each frame. On a mobile phone, standard SDF computation algorithms take many tens of milliseconds to process a single image, so they are too slow for our purposes.

The Euclidean SDF $\Phi$ of a contour is designed to increase linearly in the direction normal to the contour. This observation leads to our approximate SDF, where, for a contour point at location $\mathbf{x}$, we increase the value of $\Phi$ linearly from a value of $-d$ at location $\mathbf{x}-d \hat{\mathbf{n}}$ to a value of $+d$ at location $\mathbf{x}+d \hat{\mathbf{n}}$. Here $\hat{\mathbf{n}}$ is the normal to the contour at location $\mathbf{x}$, and is computed by applying a Scharr operator [18] to the raycast binary image. The horizontal and vertical Scharr kernels are:

$$
V=\left[\begin{array}{ccc}
+3 & +10 & +3 \\
0 & 0 & 0 \\
-3 & -10 & -3
\end{array}\right] \quad H=\left[\begin{array}{ccc}
+3 & 0 & -3 \\
+10 & 0 & -10 \\
+3 & 0 & -3
\end{array}\right]
$$

This is an approximation of the full SDF from two points of view. First, we only compute a local, per contour point $\mathrm{SDF}$, in a $\pm d$ band around the contour, as shown in Figure 2. Since the informative part of the SDF is only situated close to or on the actual contour points, this approximation has virtually no effect on the final pose optimisation result, as we show in Section 7. Second, the approximation might produce incorrect distance values around concavities of the contour, but again this did not adversely affect the final outcome of the pose optimisation.

We also need to compute the values of the derivatives $\partial \Phi / \partial x$ and $\partial \Phi / \partial y$. Numerically, these are obtained with the centred finite differences approximation, using:

$$
\frac{\partial \Phi}{\partial x}=\frac{\Phi([x+1, y])-\Phi([x-1, y])}{2}
$$

and similarly for $y$.

In this work we obtain the values of $\Phi([x+1, y]), \Phi([x-$ $1, y]), \Phi([x, y-1])$ and $\Phi([x, y+1])$ without explicitly evaluating $\Phi$. This process is represented in Figure 2. Here $\mathbf{x}_{i}$ 


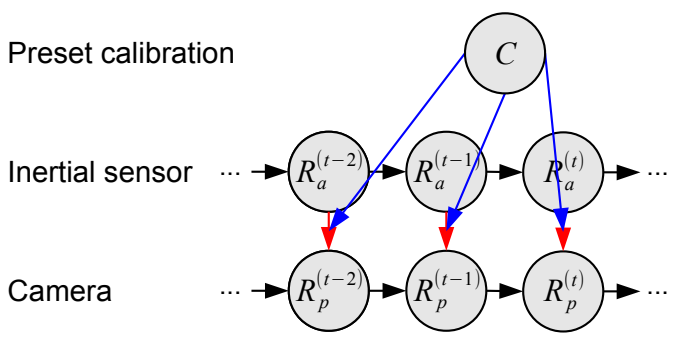

Fig. 3. Inertial sensor integration.

and $\mathbf{x}_{i+1}$ are two consecutive contour points, linked by a contour segment. Two example normals to this line segment are drawn in black, with arrows. These pass through the centre of the segment $[x, y]$ and the point $[x-1, y]$. The value of $\Phi([x-1, y])$ then is equal to the signed distance between $[x, y]$ and the projection of $[x+1, y]$ onto the normal passing through $[x, y]$. In Figure 2 this is the (signed) size of the line segment drawn in pink and bold. The process is identical for $[x+1, y],[x, y-1]$ and $[x, y+1]$.

Optimisation Method. Our raycaster produces a hierarchy of object renderings. We use this to speed up our tracker, replacing costly high resolution iterations with cheaper low resolution ones, resulting in a 2 to $3 \times$ speedup. We use the Levenberg-Marquardt (LM) algorithm to minimise the energy function at each hierarchy level.

Silhouette Ambiguity. The mapping from silhouette to pose is ambiguous, as $3 \mathrm{D}$ rigid objects often project to virtually identical silhouettes under different poses. We experimentally investigate the effect of this ambiguity on tracking in Figure 5, showing that silhouette-only tracking and reconstruction is effectively impossible. Inspired by [15], we use the inertial sensor typically available on mobile phones to disambiguate rotation.

The relation between the two pose estimations is depicted in Figure 3. $\mathrm{R}_{p}^{(t-1)}$ and $\mathrm{R}_{p}^{(t)}$ are the rotation matrices of the object in the camera coordinate system, at the previous frame and current frame, respectively. Similarly, $\mathrm{R}_{a}^{(t-1)}$ and $\mathrm{R}_{a}^{(t)}$ are consecutive rotation matrices of the camera in the inertial sensor (i.e. phone) coordinate system. Finally, $C$ is the calibration rotation matrix, converting the visual to the inertial sensor coordinate systems and is constant and precalibrated for each type of device. Therefore:

$$
\mathrm{R}_{p}^{(t)}=C \mathrm{R}_{a}^{(t)}\left(\mathrm{R}_{a}^{(t-1)}\right)^{-1} C^{-1} \mathrm{R}_{p}^{(t-1)}
$$

Between consecutive frames we only optimise for translation, using the change given by the inertial sensor as rotation estimate. To compensate for inertial sensor drift, we use one gradient descent rotation-wise iteration every ten frames. We do not use LM for rotation owing to ambiguity we only trust the visual rotation estimate to correct for slight drift, not to fully dictate the pose.

\section{Shape Optimisation}

The shape optimisation assumes known pose and per-pixel foreground or background likelihoods for each of the $n$ views. These are back-projected into a pair of 3D likelihood volumes, capturing the probability that a voxel $\mathbf{v}$ belongs to the inside and outside of the shape, respectively. The likelihoods are next turned into posteriors, in a manner similar to the one presented in the previous section. Finally, the 3D shape $u$ is extracted from the two posterior volumes, such that the inside/outside separation is maximised. This framework is similar to the one established in [8], [5], but here we use voxel posteriors instead of likelihoods and account for the online accumulation of views.

An alternative approach would have been to fuse individual per-view segmentations (obtained using, say, perview graph-cuts) instead of probabilities. This approach has been shown in [8] to produce inferior results, because (i) individual segmentations often tend to be poor (because of e.g. shadows and reflections) and (ii) the camera pose is not perfectly known, so silhouette uncertainly has to be accounted for.

Considering $\mathbf{x}$ and $\Phi$ as known in the graphical model, the joint probability for $n$ views becomes:

$$
P\left(u, \mathbf{v}, R_{1 \ldots n}, \mathbf{c}_{1 \ldots n}\right)=P\left(\mathbf{v} \mid u, R_{1 \ldots n}\right) P\left(\mathbf{c}_{1 \ldots n} \mid R_{1 \ldots n}\right) P\left(R_{1 \ldots n}\right)
$$

Expanding, we write:

$$
P\left(u \mid \Omega_{3}\right)=\prod_{\mathbf{v} \in \Omega_{3}}\left\{\sum_{j \in f, b} P\left(\mathbf{v} \mid u, R_{j, 1 \ldots n}\right) P\left(R_{j, 1 \ldots n} \mid \mathbf{c}_{1 \ldots n}\right)\right\}
$$

where $\Omega_{3}$ is the $3 \mathrm{D}$ domain of voxels $\mathbf{v}$ and:

$$
\begin{gathered}
P\left(R_{j, 1 \ldots n} \mid \mathbf{c}_{1 \ldots n}\right)=\frac{P\left(\mathbf{c}_{1 \ldots n} \mid R_{j, 1 \ldots n}\right) P\left(R_{j, 1 \ldots n}\right)}{\sum_{i \in f, b} P\left(\mathbf{c}_{1 \ldots n} \mid R_{i, 1 \ldots n}\right) P\left(R_{i, 1 \ldots n}\right)} \\
P\left(\mathbf{v} \mid u, R_{f, 1 \ldots n}\right)=\frac{u}{\zeta_{f}} \quad P\left(\mathbf{v} \mid u, R_{b, 1 \ldots n}\right)=\frac{1-u}{\zeta_{b}}
\end{gathered}
$$

with $\zeta_{f}$ and $\zeta_{b}$ being the average number of voxels (over all views $n$ ) that project to a foreground pixel (with $P\left(\mathbf{c} \mid R_{f}\right)>$ $\left.P\left(\mathbf{c} \mid R_{b}\right)\right)$ and a background pixel, respectively.

The final two probabilities $P\left(\mathbf{c}_{1 \ldots n} \mid R_{1 \ldots n}\right)$ and $P\left(R_{1 \ldots n}\right)$ should be computed as the joint probabilities of $P\left(\mathbf{c}_{k} \mid R_{k}\right)$ and $P\left(R_{k}\right)$, with $k \in 1 \ldots n$. As observed by [8], this joint probability cannot easily be computed numerically, as it tends to be a product of very small numbers. The authors in [8] used the geometric mean to obtain average probabilities. We proceed in a similar manner, and write:

$$
\begin{aligned}
& P\left(\mathbf{c}_{1 \ldots n} \mid R_{f, 1 \ldots n}\right)=\exp \left(\frac{\sum_{k=1}^{n} \log \left(P\left(\mathbf{c}_{k} \mid R_{f, k}\right)\right)}{n}\right) \\
& P\left(\mathbf{c}_{1 \ldots n} \mid R_{b, 1 \ldots n}\right)=1-\exp \left(\frac{\sum_{k=1}^{n} \log \left(1-P\left(\mathbf{c}_{k} \mid R_{b, k}\right)\right)}{n}\right)
\end{aligned}
$$

The probability of foreground and background region over $n$ views becomes $P\left(R_{f, 1 \ldots n}\right)=\overline{\eta_{f}} / \eta$ with $\overline{\eta_{f}}$ being the average value of $\eta_{f}$ (as defined in Equation (4)) over the $n$ views. $P\left(R_{b, 1 \ldots n}\right)$ follows analogously. 
The final expansion of $P\left(u \mid \Omega_{3}\right)$ becomes:

$$
\begin{aligned}
E & =P\left(u \mid \Omega_{3}\right)=\prod_{\mathbf{v} \in \Omega_{3}}\left\{u P_{i}+(1-u) P_{o}\right\} \\
P_{i} & =\frac{\overline{\eta_{f}}}{\zeta_{f}} \frac{P\left(\mathbf{c}_{1 \ldots n} \mid R_{f, 1 \ldots n}\right)}{P\left(\mathbf{c}_{1 \ldots n} \mid R_{f, 1 \ldots n}\right) \overline{\eta_{f}}+P\left(\mathbf{c}_{1 \ldots n} \mid R_{b, 1 \ldots n}\right) \overline{\eta_{b}}} \\
P_{o} & =\frac{\overline{\eta_{b}}}{\zeta_{b}} \frac{P\left(\mathbf{c}_{1 \ldots n} \mid R_{b, 1 \ldots n}\right)}{P\left(\mathbf{c}_{1 \ldots n} \mid R_{f, 1 \ldots n}\right) \overline{\eta_{f}}+P\left(\mathbf{c}_{1 \ldots n} \mid R_{b, 1 \ldots n}\right) \overline{\eta_{b}}}
\end{aligned}
$$

One way to optimise Equation (19) is to consider $u$ as the smoothed Heaviside of a 3D level set embedding function. This method is subject to local minima and requires the SDF structure to be maintained. When $u$ is a 3D volume of probabilities, [10] shows that $u$ can be solved for globally using convex optimisation.

The globally solvable formulation for our reconstruction is obtained by replacing the logarithmic opinion pool with a linear one in Equation (14) and adding a weighted surface regularization term:

$$
E=\sum_{\mathbf{v} \in \Omega_{3}}\left\{u P_{i}+(1-u) P_{o}+\alpha|\nabla u|\right\}
$$

where $\alpha$ is a tunable parameter.

To minimise such an energy function the authors in [8] use the primal-dual algorithm of [12]. We use the continuous min-cut / max-flow formulation of [25], which leads to considerably faster convergence compared to [12]. In a max-flow context, minimising Equation (19) with respect to $u$ such that $u \in[0,1]$ is equivalent to minimising:

$$
E=\max _{p_{t}, p_{s}, p} \min _{u} \sum_{\mathbf{v}}\left\{u p_{t}+(1-u) p_{s}+u \operatorname{div} p\right\}
$$

such that $p_{s}(\mathbf{v})<P_{i}(\mathbf{v}), p_{t}(\mathbf{v})<P_{o}(\mathbf{v})$ and $|p(\mathbf{v})|<\alpha$. In the context of max-flow, $p, p_{s}$ and $p_{t}$ are flow capacities, for undirected edges for $p$, between nodes and source for $p_{s}$ and between nodes and sink for $p_{t}$ [25].

Continuous max-flow [25] is an iterative algorithm that uses the augmented Lagrangian function of Equation (23):

$$
\begin{aligned}
& L_{c}\left(p_{s}, p_{t}, p, u\right)= \\
& \quad \sum_{\mathbf{v}}\left\{u p_{t}+(1-u) p_{s}+u \operatorname{div} p\right\}-\frac{c}{2}\left\|\operatorname{div} p-p_{s}+p_{t}\right\|^{2}(24)
\end{aligned}
$$

where $c$ is a constant step size. This Lagrangian is used to minimise Equation (23) wrt. $p, p_{s}$ and $p_{t}$ in turn. For the $l$-th iteration of the algorithm the authors in [25] write:

$$
\begin{aligned}
& p^{(l+1)}=\underset{\|p\|_{\infty} \leq \alpha}{\arg \max } L_{c}\left(p_{s}^{l}, p_{t}^{l}, p, u^{l}\right) \\
& p_{s}^{(l+1)}=\underset{p_{s}(\mathbf{v})<P_{i}(\mathbf{v})}{\arg \max } L_{c}\left(p_{s}, p_{t}^{l}, p^{l}, u^{l}\right) \\
& p_{t}^{(l+1)}=\underset{p_{t}(\mathbf{v})<P_{o}(\mathbf{v})}{\arg \max } L_{c}\left(p_{s}^{l}, p_{t}, p^{l}, u^{l}\right) \\
& u^{(l+1)}=u^{(l)}-c\left(\operatorname{div} p^{(l+1)}-p_{s}^{(l+1)}+p_{t}^{(l+1)}\right)
\end{aligned}
$$

The optimisation is started by setting $u=P_{i}-P_{o}$ where $P_{i}>P_{o}$ and zero otherwise, and $p_{s}=p_{t}=\min \left(P_{i}, P_{o}\right)$.

So far we have assumed that all $n$ views are available simultaneously. This is not true in our case, as we run our algorithm online. We therefore propose four changes to the reconstruction methodology.
First, the likelihood volumes $P\left(\mathbf{c}_{1 \ldots n} \mid R_{1 \ldots n}\right)$ and the values of $\zeta_{f}, \zeta_{b}, \overline{\eta_{f}}$ and $\overline{\eta_{b}}$ are updated continuously online instead of accumulated only once for all $n$ views.

Second, it is often the case that tracking with an inaccurate shape leads to inaccurate pose results. This means that, primarily in the beginning of the reconstruction process, we pollute the joint foreground likelihoods $P\left(\mathbf{c}_{1 \ldots n} \mid R_{f, 1 \ldots n}\right)$ with pixels that actually belong to the background, and vice-versa. Tracking does gradually improve as the shape becomes more accurate, but colour likelihoods have already been incorrectly integrated. A solution to this problem would be to maintain a history of the likelihood changes as a function of pose. When observing the target a second time from any given pose we would replace previously integrated likelihoods from the probability volume and with new ones. Such an approach is however not tractable on a mobile phone due to memory constraints. Here we use an alternative, in the form of a sliding average. Assuming $n$ already registered views, for a new view $n+1$ we write:

$$
\begin{aligned}
& P\left(\mathbf{c}_{1 \ldots n} \mid R_{f, 1 \ldots n+1}\right)= \\
& \exp \left(\frac{w \sum_{k=1}^{n} \log \left(P\left(\mathbf{c}_{k} \mid R_{f, k}\right)\right)+\log \left(P\left(\mathbf{c}_{n+1} \mid R_{f, n+1}\right)\right)}{w+1}\right)
\end{aligned}
$$

where $w$ is capped to a fixed value (e.g. 250). $P\left(\mathbf{c}_{1 \ldots n} \mid R_{b, 1 \ldots n+1}\right)$ follows analogously.

Third, after running the max-flow algorithm, we rescale the non-zero region of interest in the 3D volume $u$ to fill the volume, with a constant padding. This mitigates the effects of scale drift and helps us use the full representational power of the discretisation.

Fourth, instead of running the continuous max-flow optimisation to full convergence after all $n$ views have been registered, we run a single iteration for every five views that have been registered. We run only one iteration for real-time considerations as the final estimate will change with updated input data anyway. This leads to another problem, namely how to transfer the intermediate shape results from one reconstruction to the next. This is not straightforward, as, especially in the early stages, large portions of the estimated 3D shape might change and the intermediary values for $u$ are far from the globally optimal shape embedded by the updated posterior volumes.

To alleviate this problem we propagate the intermediary values of the 3D shape by conditioning $P\left(\mathbf{v} \mid u, R_{f, 1 \ldots n}\right)$ and $P\left(\mathbf{v} \mid u, R_{b, 1 \ldots n}\right)$ on the previous 3D reconstruction $u^{(t-1)}$. Intuitively, this means that the probabilities of a voxel being inside or outside the 3D shape should increase if the voxel was inside or outside, respectively, at the previously available reconstruction. Formally this changes:

$$
\begin{aligned}
& P\left(\mathbf{v} \mid u, R_{f, 1 \ldots n}\right) \text { to } P\left(\mathbf{v} \mid u, u_{i}^{(t-1)}, R_{f, 1 \ldots n}\right)=\frac{u u_{f}^{(t-1)}}{\zeta_{f}} \\
& P\left(\mathbf{v} \mid u, R_{b, 1 \ldots n}\right) \text { to } P\left(\mathbf{v} \mid u, u_{o}^{(t-1)}, R_{b, 1 \ldots n}\right)=\frac{(1-u) u_{b}^{(t-1)}}{\zeta_{b}}
\end{aligned}
$$


with:

$$
u_{i}^{(t-1)}=\beta-1+\beta u^{(t-1)} \quad u_{o}^{(t-1)}=\beta-1+\beta\left(1-u^{(t-1)}\right)
$$

where $\beta$ is a tunable parameter ( 0.5 in our implementation).

The change in voxel probabilities also leads to slightly different formulas $P_{i}$ and $P_{o}$, which are trivial to compute.

\section{IMPLEMENTATION AND TIMINGS}

Next we present a practical implementation of the theoretical framework introduced above. We dissociate the tracking and reconstruction tasks and run them in parallel as two separate threads, in a manner akin to [7]. Sections 6.1 and 6.2 present the implementation of the two threads and Section 6.3 shows timing results.

\subsection{Tracking Thread}

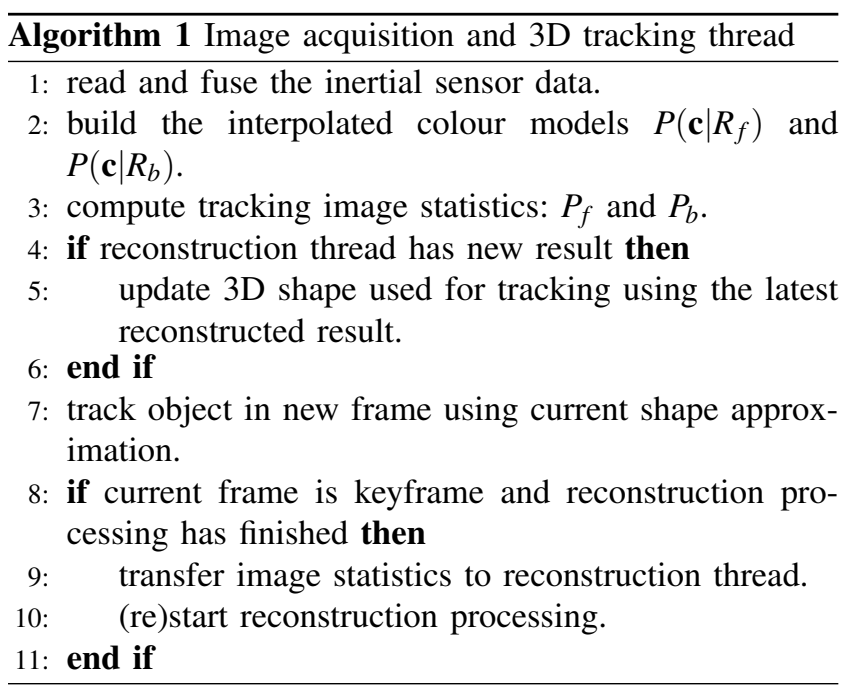

The implementation of the tracking thread is detailed in Algorithm 1. This is executed every time a new frame is available from the camera.

The first step is to compute the orientation of the device from the inertial sensor measurements. We used Apple iPhones throughout our experiments, so this step is done using the Apple Core Motion framework and the fused gyroscope and accelerometer sensor measurements.

Next, we must extract the image statistics (i.e. the pixel wise foreground and background posterior probabilities $P_{f}$ and $P_{b}$ ), used by both the reconstruction and tracking stages. This requires known foreground and background colour models $P\left(\mathbf{c} \mid R_{f}\right)$ and $P\left(\mathbf{c} \mid R_{b}\right)$, which in our case are 32 bin RGB histograms. A common approach [2], [14] is to initialise the models using a known single imagemask pair and adapt them online using the instantaneous object segmentation. This approach fails in our case as the histograms are quickly polluted in the beginning of the reconstruction process. Our solution is to capture several ( 5 to 10) colour models using image-mask pairs taken from various positions around the object, along with their associated inertial sensor rotation measurements. At runtime, for each novel frame, we build a set of pose dependant colour models, using linear interpolation on the set of histogram initialisations, weighted by the distance in rotation space between the instantaneous inertial sensor measurement and the pre-captured ones. The initial object segmentations are done very easily by the user, by keeping the object centred and adjusting a slider which changes the threshold value of a binarisation algorithm. Alternative approaches, such as saliency, could be used.

The next major step is update the $3 \mathrm{D}$ position of the camera on the new frame using the newly obtained image statistics. Before doing this however, we update the 3D volume used for tracking with the latest approximation of the object shape built by the reconstruction thread. We use $64 \times 64 \times 64$ volumes for tracking and $128 \times 128 \times 128$ for reconstruction, so this stage also involves a trilinear rescaling. As described in Section 4, we use the LevenbergMarquardt algorithm to track the camera. The method used to compute the pose derivative is outlined in Algorithm 2.

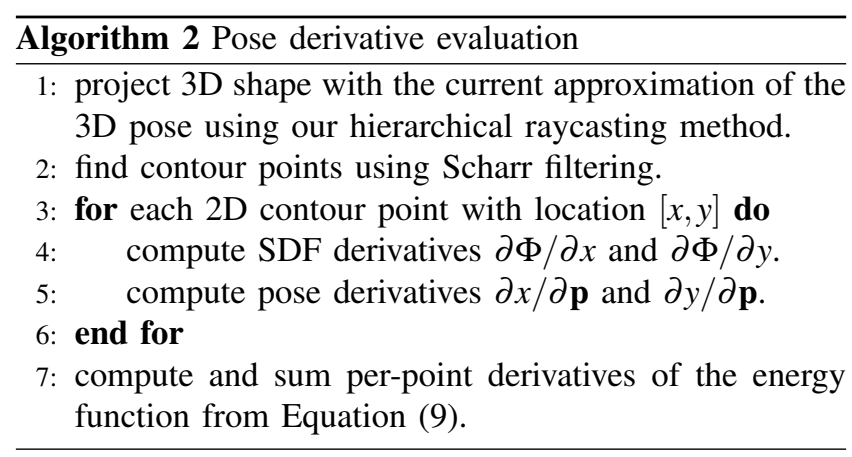

Once the current camera pose has been computed, the image statistics are transferred to the reconstruction thread. This process is keyframe based, with keyframes at least three frames apart and with an inertial sensor rotation observed a maximum of five times. We allow each rotation several times to accommodate updates due to improved pose estimates. We could have followed the more traditional approach of using the estimated translation, but experimentally we found our solution to lead to better results.

\subsection{Reconstruction Thread}

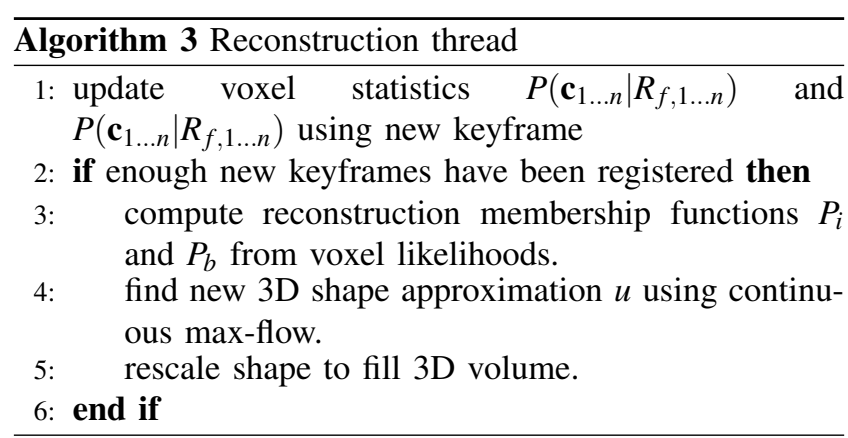

The reconstruction thread is described as Algorithm 3. We first update the voxel statistics $P\left(\mathbf{c}_{1 \ldots n} \mid R_{f, 1 \ldots n}\right)$ and $P\left(\mathbf{c}_{1 \ldots n} \mid R_{f, 1 \ldots n}\right)$ using the pixel statistics from the new 
TABLE 1

Per frame example timings for our method.

\begin{tabular}{|l|c|c|c|}
\hline & Tracking & Frame registration & Max flow \\
\hline Desktop PC & $9.3 \mathrm{~ms}$ & $18 \mathrm{~ms}$ & $102 \mathrm{~ms}$ \\
\hline iPhone 5 & $43.3 \mathrm{~ms}$ & $198 \mathrm{~ms}$ & $667 \mathrm{~ms}$ \\
\hline iPhone 5s & $31.7 \mathrm{~ms}$ & $143 \mathrm{~ms} \mathrm{CPU} \mathrm{/} \mathrm{88ms} \mathrm{GPU}$ & $504 \mathrm{~ms}$ \\
\hline
\end{tabular}

keyframe. This process is implemented on the mobile phone GPU using a vertex shader and the OpenGL transform feedback. The voxel location is sent as a parameter to the shader which computes the per-frame foreground background likelihoods and writes them back to CPU accessible memory, where the sliding average method is used to produce the new set of joint colour likelihoods.

After a sufficiently large number of novel keyframes has been accumulated (five in our case), we compute the inside/outside membership functions from the voxel statistics and use a vanilla implementation of the continuous max-flow algorithm [25] to update the 3D shape $u$. The last step is to centre and rescale the useful areas of the update 3D shape (where $u>0.5$ ) to fill the reconstruction volume.

\subsection{Timings}

Table 1 shows example timings obtained by our method for the sequence shown later in Figure 13. The tracker runs at $107 \mathrm{fps}$ on a desktop PC (Intel Core i7-3960X 3.3GHz CPU), $23 \mathrm{fps}$ on an iPhone 5 and $31 \mathrm{fps}$ on an iPhone 5 s. Performance degrades roughly $25 \%$ on an iPhone $4 \mathrm{~s}$. This means that tracking is possible on lower end devices but reconstruction is not. GPU-based frame registration is much faster than the CPU version, but our implementation is only possible on the iPhone $5 \mathrm{~s}$ (as it uses the transform feedback capability introduced in OpenGL ES 3.0).

Tracking is run at each frame, the frame registration every 3rd frame and the continuous max-flow optimisation once five keyframes have been accumulated. Our method can run on a continuous stream of novel frames, or, once a critical mass of frames has been obtained (in our experiments 1500 frames) we can re-track and re-register previous ones to refine the model in a postprocessing step.

\section{Results}

We have tested our method quantitatively and qualitatively, on both artificial and real data, in static and dynamic environments.

Quantitative Testing. We begin in Figure 4 with a quantitative comparison between the PTAM system [7], our tracker and the one presented in [16], which minimises the same energy function in a fully analytical manner, without any approximations. Note that in all the experiments containing charts, the colour assigned to the model corresponds to the colour of the chart plot. The results produced by the object trackers are nearly identical. The average differences in translation and rotation between [16] and PTAM are 9.6 $\mathrm{mm}$ and $2.0^{\circ}$ and between this work and PTAM are 11.3 $\mathrm{mm}$ and $2.5^{\circ}$. The small improvement in the tracker from
[16] comes however as the expense of twice the processing time and the added requirement of powerful GPU.

Figure 5 shows a quantitative comparison between our tracker with and without using the inertial sensor and PTAM [7] for another sequence. Without the inertial sensor, the average difference between tracker and PTAM is 129 $\mathrm{mm}$ and $12.0^{\circ}$. With the inertial sensor the difference decreases to an average of $11.4 \mathrm{~mm}$ and $1.3^{\circ}$. The very large difference incurred when the inertial sensor is not available is due to silhouette ambiguity, as shown in columns 2,4 and 6 of Figure 5(top). The silhouette provides too little information for an accurate rotation estimation and the optimisation converges to a incorrect local minima. When aided by the inertial sensor, rotation is no longer ambiguous and the overall tracking errors become much smaller.

Note that in both of the above tests, the difference between the trackers and PTAM is caused by the formers' use of information from only around the projected contour, and the latter's use of the whole image. Note that, while the PTAM system is more likely to be closer to the ground truth, it is not guaranteed to be the actual ground truth.

Figure 6 shows a quantitative comparison between the reconstruction obtained using the posterior voxel probabilities presented in this paper and the likelihoods used in [8], which we consider as close to or at the current state-of-theart. Here we generate artificial ground truth data using the human body shown in Figure 6. The generated sequence shows the $3 \mathrm{D}$ shape rotated on each axis between $\pm 180^{\circ}$ and translated by a random amount. We ran the reconstruction algorithm using the two types of voxel statistics with four configurations: (i) ground truth histograms and known pose (Figure 6 - left chart); (ii) ground truth histograms and camera rotation with optimisation for translation (Figure 6 - left chart); (iii) noisy histograms and known pose (Figure 6 - right chart) and (iv) noisy histograms, known rotation + optimisation for translation (Figure 6 - right chart). The red contour in the lower left sub-figure shows the area of foreground that was added to the estimation of the background histogram in order to corrupt it. The matching score plotted in the four charts is the standard intersection vs reunion measure of overlap [6], evaluated between ground truth and reconstructed volume. This is plotted against the number of continuous max-flow iterations (i.e. number of registered keyframes times five). Note that we do not run any shape alignment since all versions of the algorithm should produce aligned shapes.

When the histograms are not noisy all four methods produce similar results, with the best ones being obtained using posteriors and ground truth poses. Overall, likelihoods produce a less accurate discrimination between foreground and background. In the beginning of the reconstruction process this has the advantage that more of the shape is left uncut, so tracking is more stable. This may lead to better reconstruction results, as visible in the early part of the reconstruction process depicted in Figure 6 - left chart. As soon as the reconstructed shape begins to stabilise, the posteriors lead to superior results. The difference between posteriors and likelihoods becomes much more pronounced 

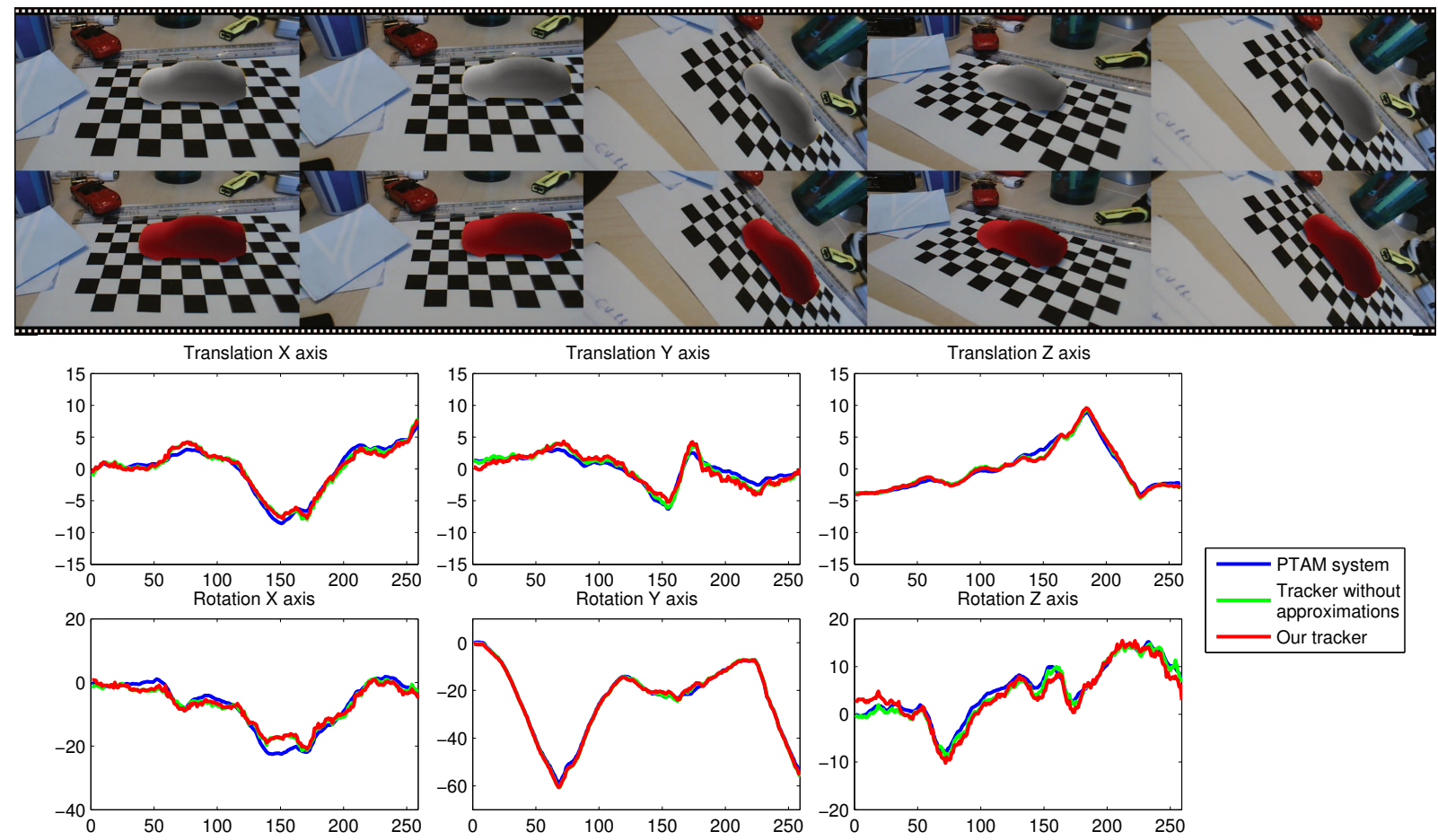

Fig. 4. Comparison between the camera pose recovered by our tracker, the tracker of [16] and the PTAM system of [7] as ground truth. The filmstrips show frames from the experiment, with the top row showing our results and the bottom row the results from [16].

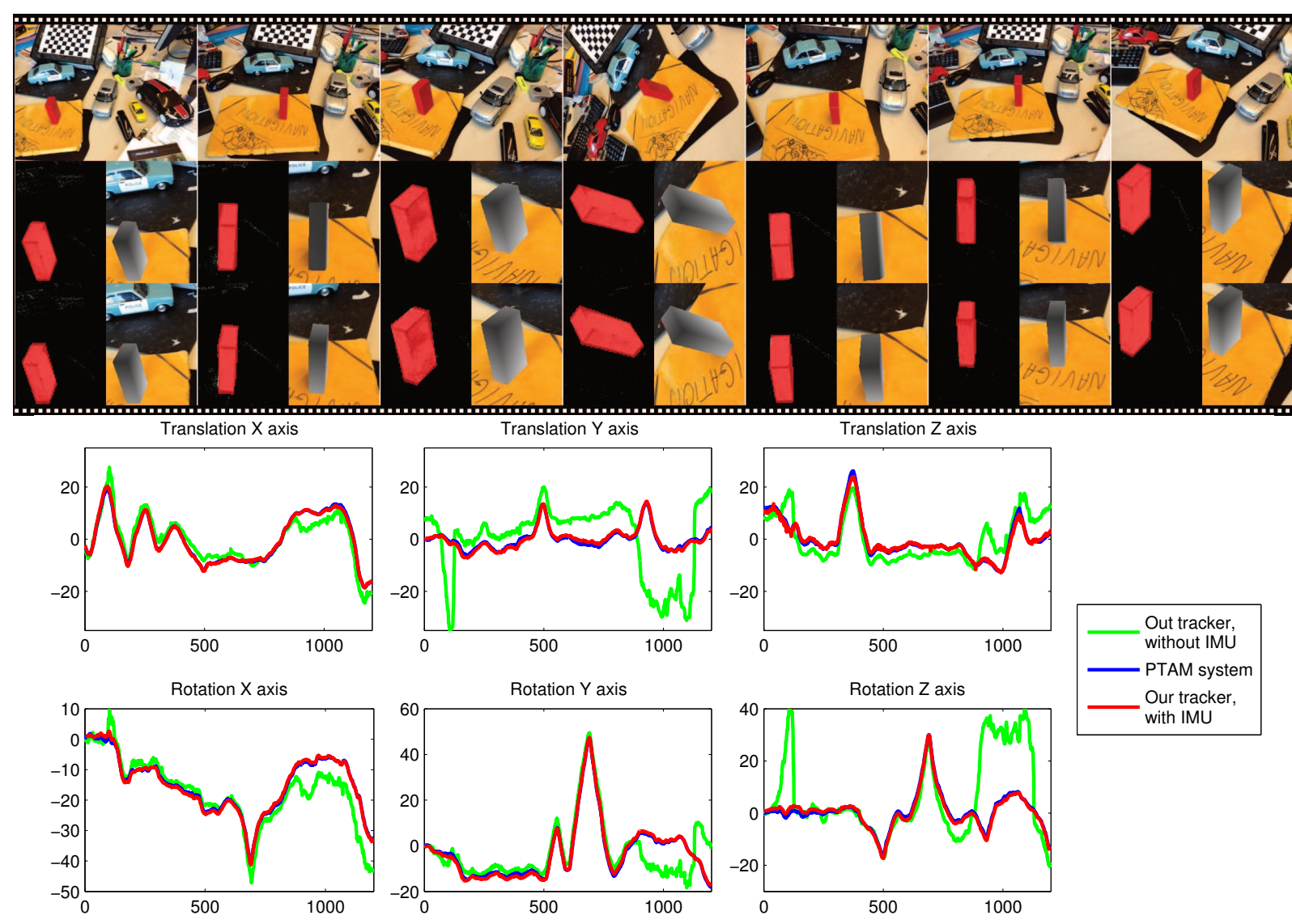

Fig. 5. Comparison between our tracker with and without the inertial sensor and the PTAM system of [7] as ground truth. The filmstrip shows frames from the experiment with row 1 showing the original image, row 2 the result obtained without the inertial sensor and row 3 the results obtained with the inertial sensor. 

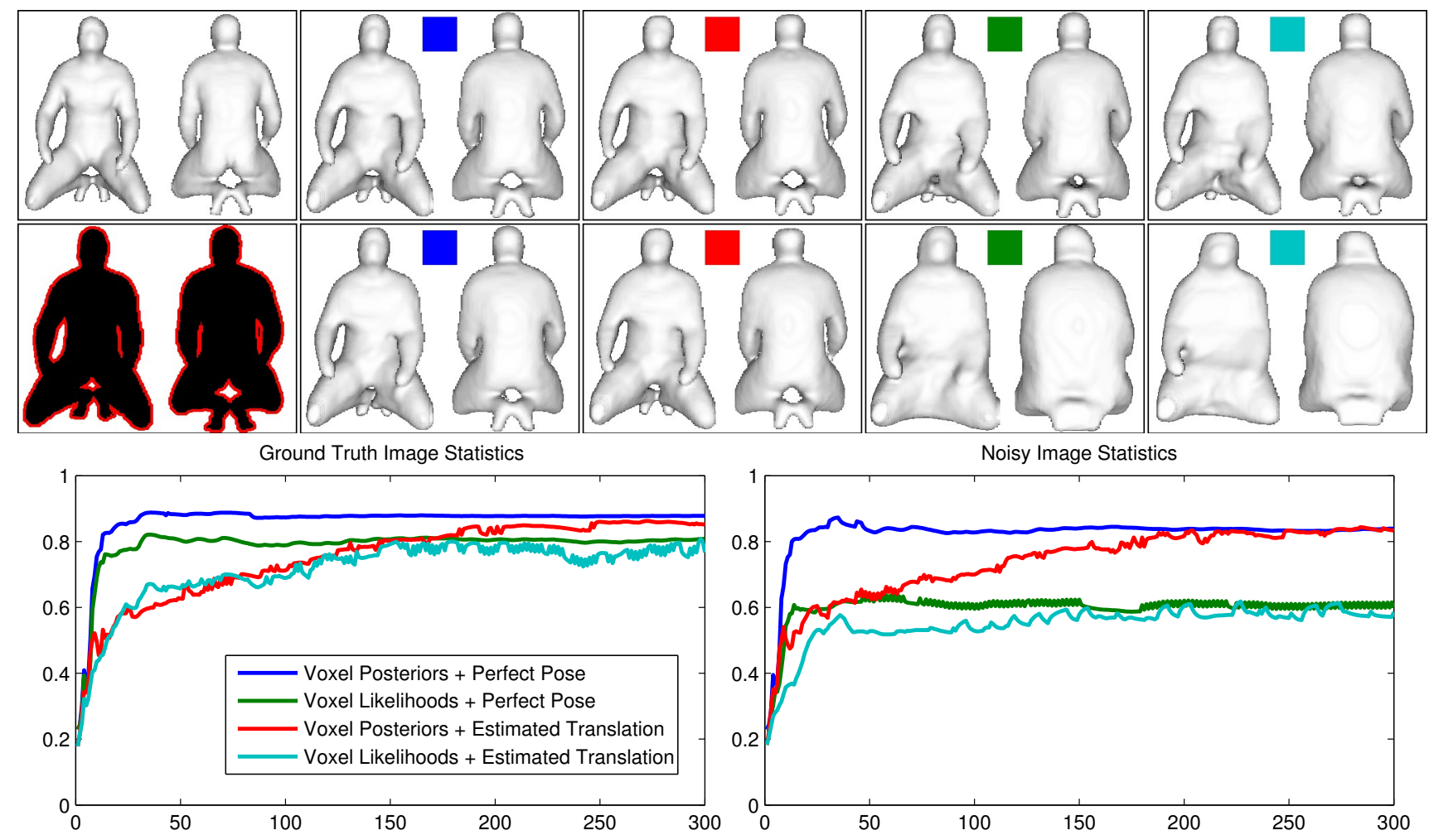

Fig. 6. Reconstruction comparison between our system and that of [8] on a crouching human form. The top 2 rows show views of the 3D reconstructed shape, with the lower left figures showing in red the foreground area that was added to the background histogram to corrupt it. Results obtained with ground truth histograms appear in the left chart and top row and results obtained with imperfect histograms in the right chart and bottom row.
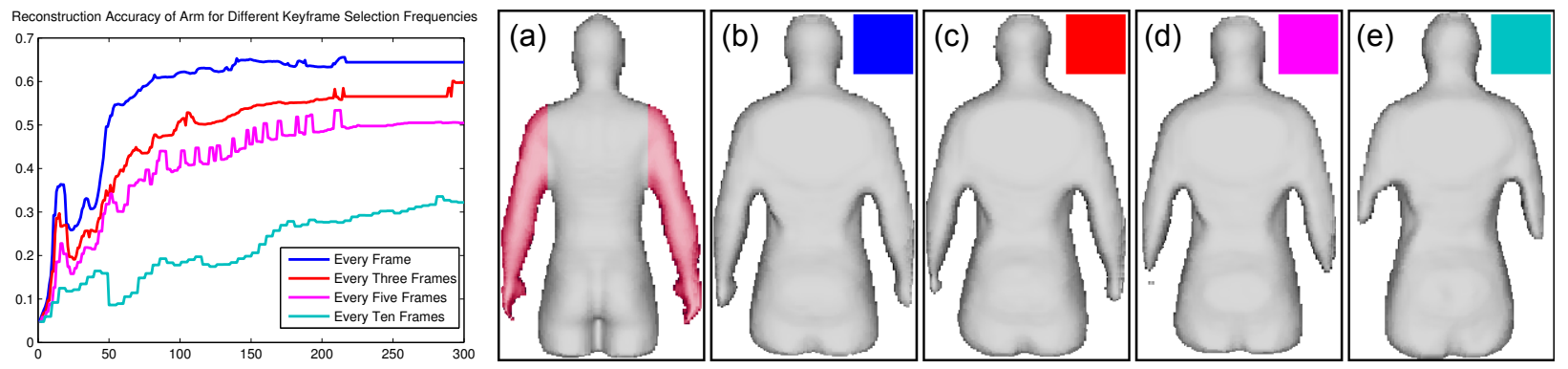

Fig. 7. Comparison between the reconstruction accuracy obtained for the arms of the person represented by the 3D shape at various keyframe selection frequencies: (a) - ground truth and (in red) the regions of the model used for the comparison; (b,c,d,e) - final reconstructions when allowing the keyframes to be 1,3,5 and 10 frames apart, respectively.
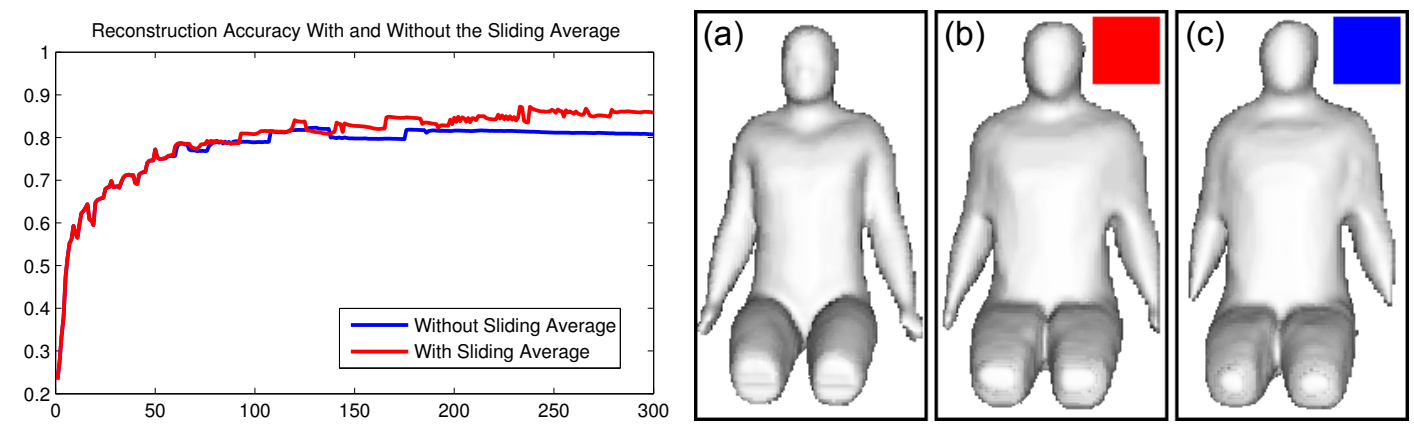

Fig. 8. Comparison between the reconstruction accuracy obtained for (a) the ground truth shape, (b) when the sliding average is used and (c) not used. 
with imperfect histograms, as is often the case in real world usage. Here using likelihoods leads to many shape details being lost and to a decrease in accuracy of over $20 \%$.

Figure 7 shows the reconstruction accuracy depending on the sampling frequency of keyframes. Registering keyframes more often leads to faster convergence. The best results are obtained when keyframes are allowed to be a single frame apart, with the accuracy decreasing as larger separations between keyframes are imposed. In the earlier version of this work, we were limited by the speed of the implementation to a minimum of ten frames between keyframes. In this work, as the implementation is now considerably faster, we can sample three times as often i.e. allow keyframes to be three frames apart, while still running in real time on a mobile phone.

Figure 8 shows how using the sliding average affects the reconstruction accuracy. Computing a full average means that the colour likelihoods registered in the beginning of the reconstruction process are weighted equally to the ones registered in the later parts of the this process. This is incorrect, as the initial inaccurate shape leads to inaccurate pose tracking results. This means that background likelihoods are registered as foreground and vice-versa. The sliding average allows us to weight early measurements less than recent ones, which improves performance.

Qualitative Testing. The following five figures (9, 10, 11,12 and 13) show qualitative reconstruction results obtained on real data. In each figure, the first three columns of the film strip show the beginning of the reconstruction process and are taken within the first 50 processed keyframes. The next two columns show results obtained around the 200-300 keyframe mark. The last column shows the final result obtained after a maximum of 1000 keyframes. Figures 9 and 10 show our reconstruction working in a museum, in a fully unstructured and dynamic environment, corrupted by occlusions, reflections and imperfect illumination. Figure 9 also shows the textured end result, while Figure 10 shows a 3D printed replica of the final result. Our method achieves good performance, with artefacts appearing only around the head of each figure. These are caused by specular reflections, which changed the colour of the object from red and black respectively to white. To our knowledge, no other object reconstruction method could process these videos because of the large number of image imperfections. Figures 11, 12 and 13 show reconstruction results obtained in a slightly better lit office environment, with Figure 11 also showing the textured result and 12 the 3D printed replica. In all three cases we can obtain very good reconstructions, with the shape usually converging within 300 to 400 keyframes (i.e. 1-2 minutes of video data).

Failure Cases. Figure 14 showcases a typical failure case for our method. Our two main sources of error are the use of (i) silhouettes and (ii) histograms as colour models. The former means that we are unable to recover the parts of the shape that are not visible along the silhouette. Examples are the indentation of the hat in Figure 14 and the inside of the shoe in Figure 13. The latter means that image artefacts such as specularities and shadows decrease the reconstruction accuracy. This leads to increased thickness in the base of reconstructed hat in Figure 14, and to the incorrect indentation in the head of the lion in Figure 9.

The other primary failure case present in [13], namely our brittleness when dealing with thin structures, has been addressed by our more frequent use of keyframes and the sliding average. This however remains a problem and our method still has trouble reconstructing very thin parts of the object (e.g leaves, fingers). Another possible source of failure in our system is rotation drift in the inertial sensor. Limited amounts of drift are compensated for using the visual rotation optimisation, but large amounts of drift result in decreased tracking and subsequently rotation accuracy.

\section{Conclusions}

We have presented a novel framework for simultaneous 3D tracking and reconstruction of objects that runs in real time on mobile phones.

Tracking is formulated as the minimisation of a region based level set energy function wrt. the 3D pose of the object. The energy function uses foreground and background appearance models, and the goal is to maximise the separation between these two regions induced by the projection of the 3D object.

Evaluating and minimising such an energy typically requires the computation of a signed distance transform and its derivative, but we present an alternative formulation which avoids this costly computation, and show that this is one of the major contributing factors towards the efficiency of our method. Further speedups are achieved using a resolution hierarchy in the raycasting step.

The energy function has inherent ambiguities for $3 \mathrm{D}$ pose estimation, but we demonstrate that these can be resolved by using additional rotation information from the inertial sensor found in modern mobile phones. Overall this results in our tracker achieving framerates of more than $100 \mathrm{~Hz}$ on a desktop PC and better than $30 \mathrm{~Hz}$ on the best mobile devices, both without involving a GPU.

Reconstruction of the 3D object model occurs simultaneously in a parallel thread. We define probabilities of lying inside or outside the object in a $3 \mathrm{D}$ volume, and re-estimate these using a continuous max-flow algorithm. The use of posterior voxel probabilities improves robustness and accuracy, particularly with imperfect image data. By moving trivially parallelisable parts of these computations onto the simple GPUs available on modern mobile phones, we manage to run the reconstruction step at $11 \mathrm{fps}$, allowing the integration of more information in the $3 \mathrm{D}$ volume and leading to faster convergence and increased accuracy.

The main direction for future research is the incorporation of texture information along with the current region based method.

\section{ACKNOWLEDGMENT}

This work was supported in Oxford by EPSRC grants EP/H050795 and EP/J014990, and by EU FP7 grant 287713 (REWIRE) and in Adelaide by the award of an Australian 


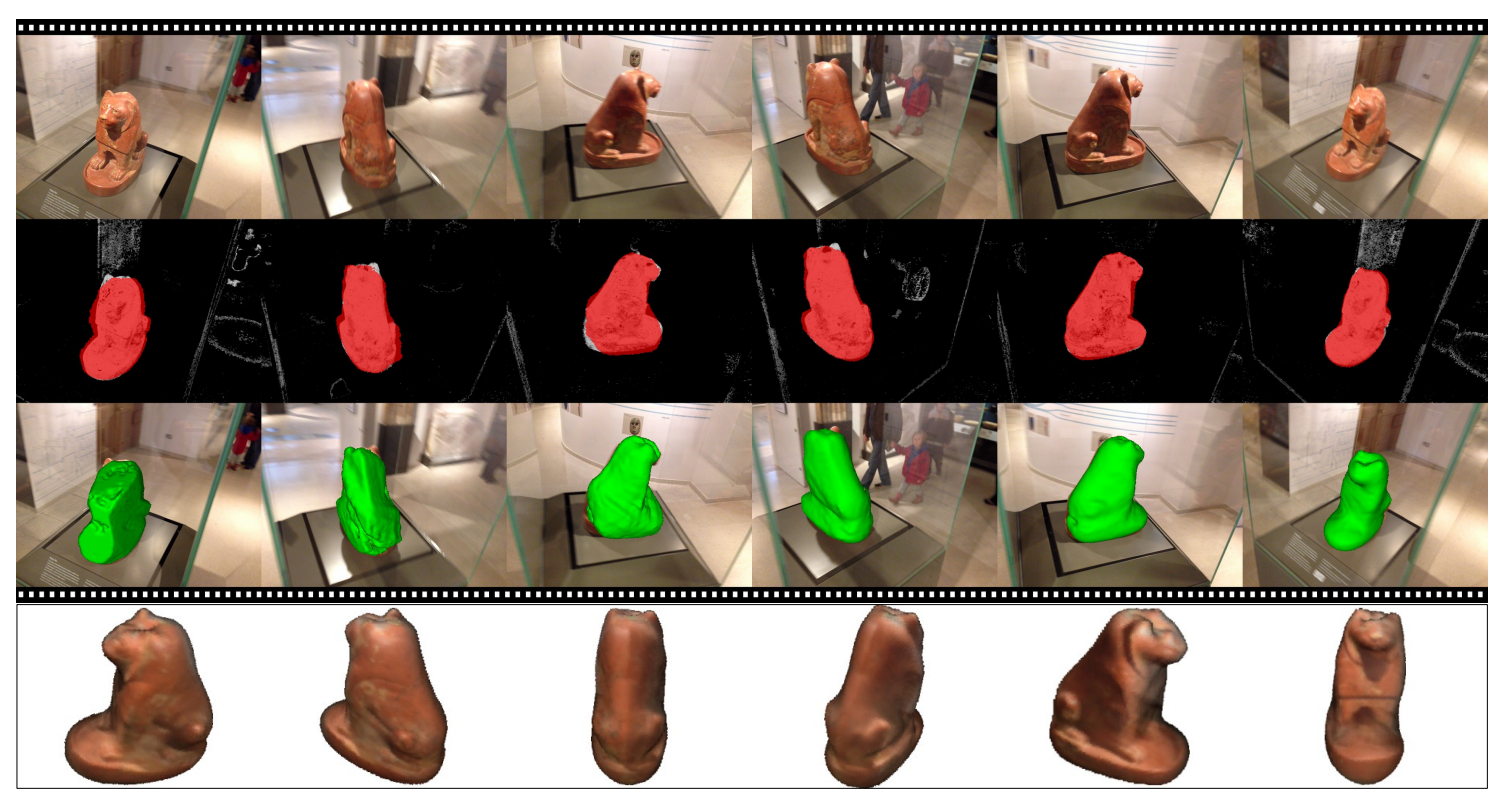

Fig. 9. Lion tracking and reconstruction example. The subject is within a reflective glass case.

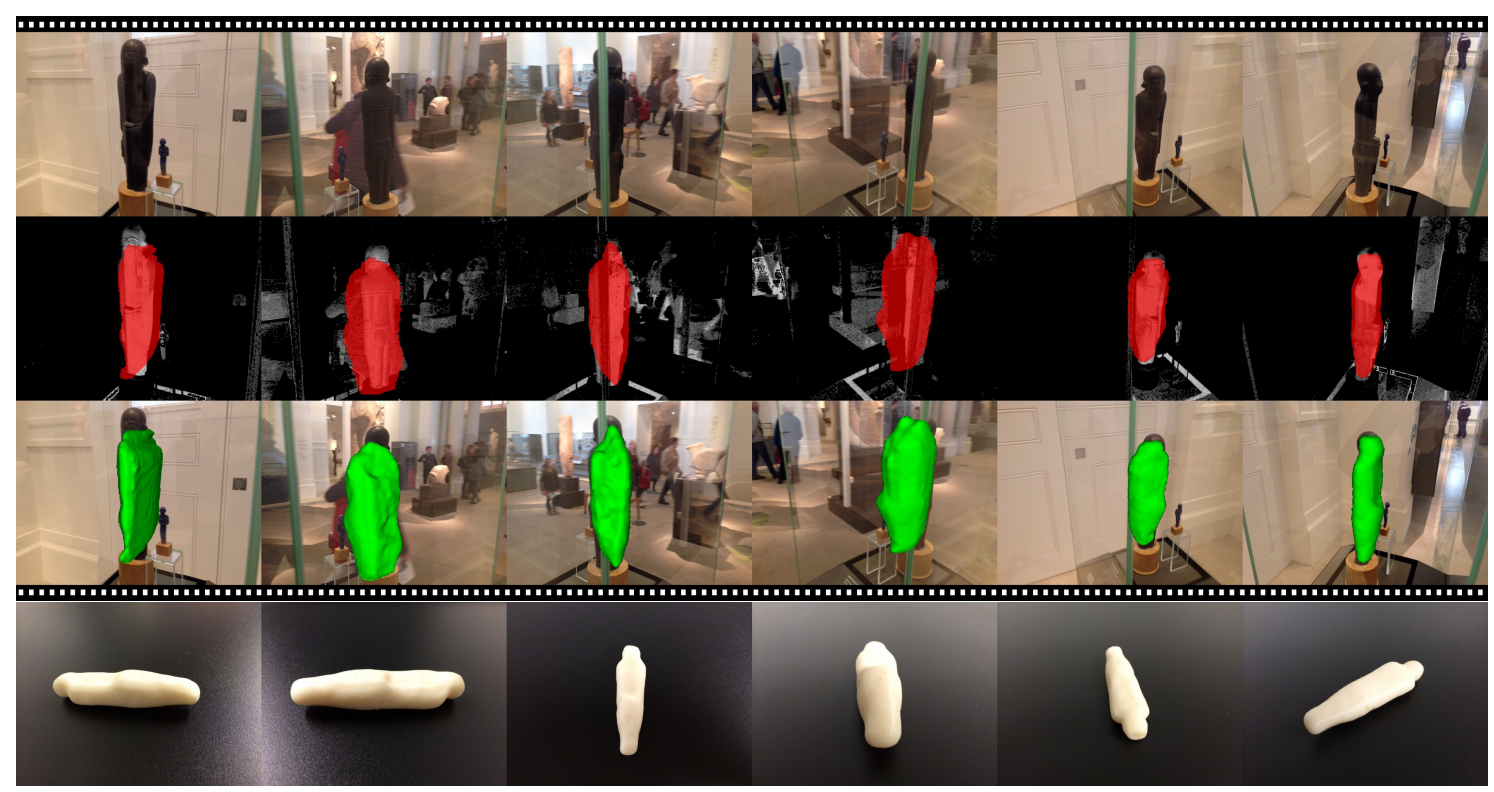

Fig. 10. Idol tracking and reconstruction example. The subject is within a reflective glass case.

Research Council Laureate Fellowship FL130100102 to Ian Reid.

\section{RefEREnCES}

[1] J. Bastian, B. Ward, R. Hill, A. van den Hengel, and A. Dick. Interactive modelling for AR applications. In ISMAR 2010, pages 199-205.

[2] C. Bibby and I. Reid. Robust Real-Time Visual Tracking Using Pixel-Wise Posteriors. In ECCV 2008, pages 831-844.

[3] G. Bleser, C. Wohlleber, M. Becker, and D. Stricker. Fast and stable tracking for ar fusing video and inertial sensor data. In WSCG 2006, pages $109-115$.

[4] N. D. Campbell, G. Vogiatzis, C. Hernández, and R. Cipolla. Automatic object segmentation from calibrated images. In CVMP 2011, pages 126-137.

[5] N. D. F. Campbell, G. Vogiatzis, C. Hernández, and R. Cipolla. Automatic 3D object segmentation in multiple views using volumetric graph-cuts. ImaVis 2010, 28(1):14-25.
[6] M. Everingham, L. van Gool, C. Williams, J. Winn, and A. Zisserman. The Pascal Visual Object Classes (VOC) Challenge. IJCV 2010, 88(2):303-338.

[7] G. Klein and D. Murray. Parallel Tracking and Mapping for Small AR Workspaces. In ISMAR 2007, pages 1-10.

[8] K. Kolev, T. Brox, and D. Cremers. Fast Joint Estimation of Silhouettes and Dense 3D Geometry from Multiple Images. IEEE Trans. on PAMI 2012, 34(3):493-505.

[9] R. A. Newcombe, S. J. Lovegrove, and A. J. Davison. DTAM: Dense tracking and mapping in real-time. In ICCV 2011, pages 2320-2327.

[10] M. Nikolova, S. Esedoglu, and T. Chan. Algorithms for Finding Global Minimizers of Image Segmentation and Denoising Models. SIAM-JAM 2006, 66(5):1632-1648.

[11] Q. Pan, G. Reitmayr, and T. Drummond. ProFORMA: Probabilistic Feature-based On-line Rapid Model Acquisition. In BMVC 2009, pages $1-11$.

[12] T. Pock, D. Cremers, H. Bischof, and A. Chambolle. An Algorithm for Minimizing the Piecewise Smooth Mumford-Shah Functional. In ICCV 2009, pages 1133-1140. 


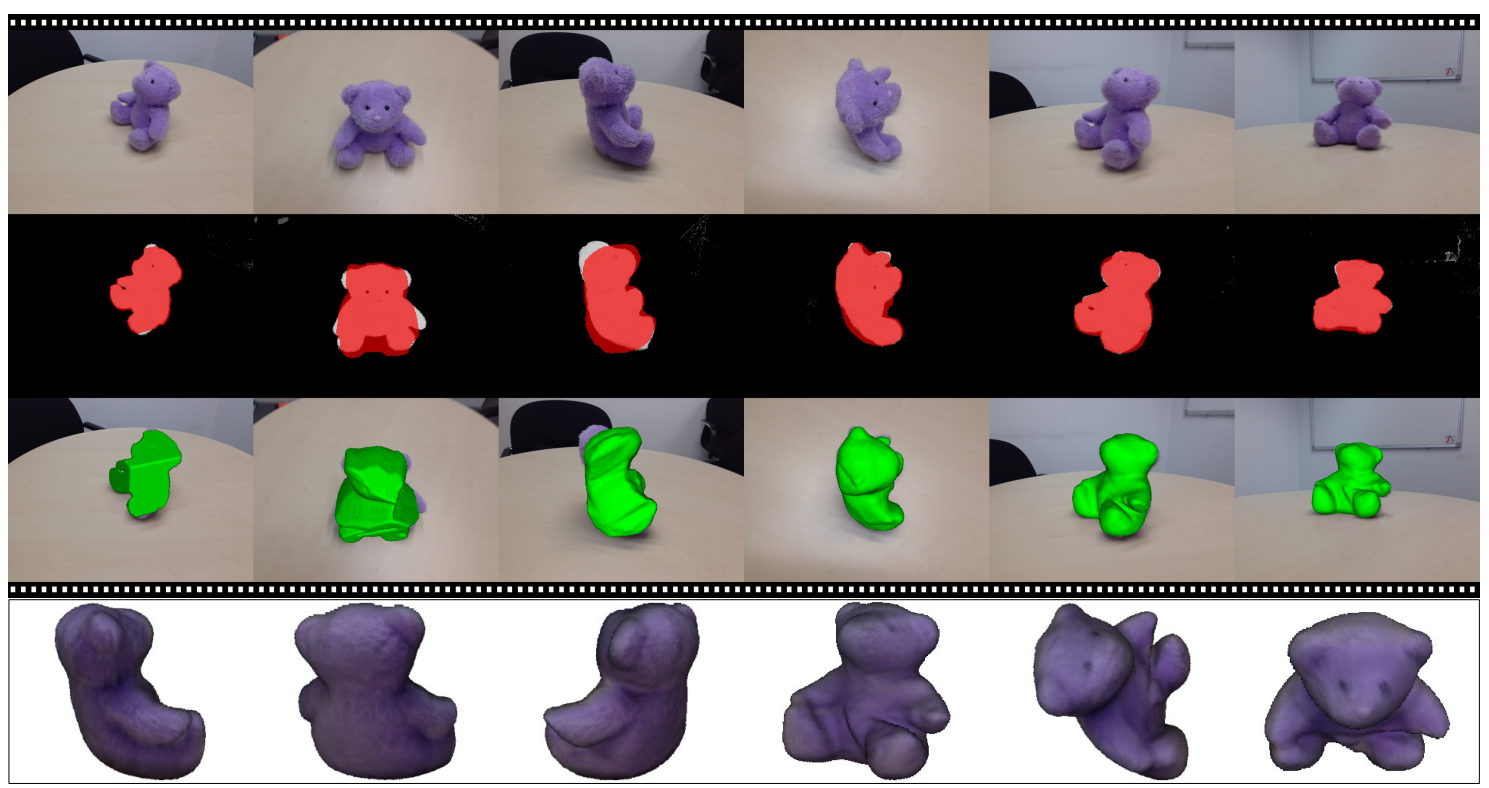

Fig. 11. Teddy bear tracking and reconstruction example.
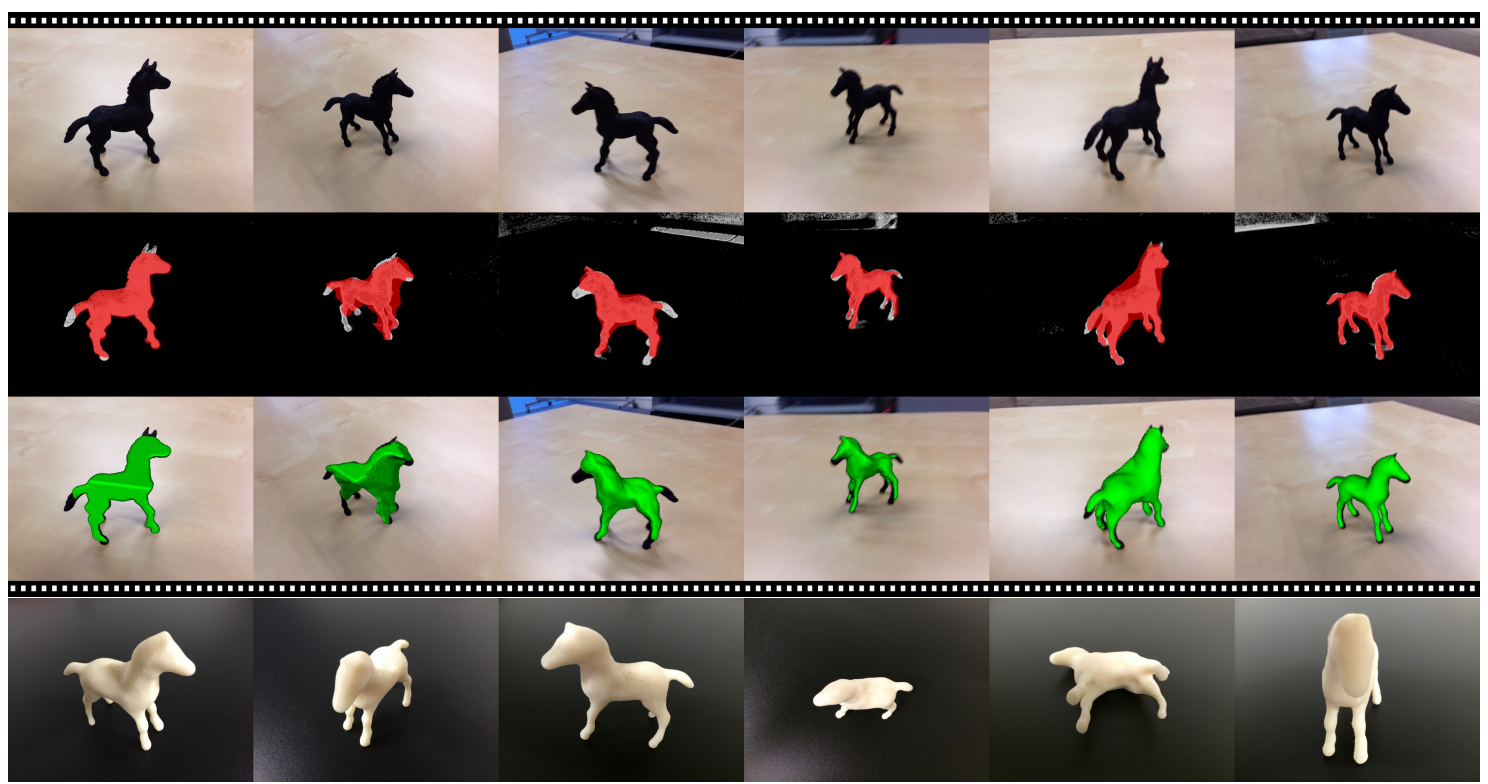

Fig. 12. Toy horse tracking and reconstruction example.

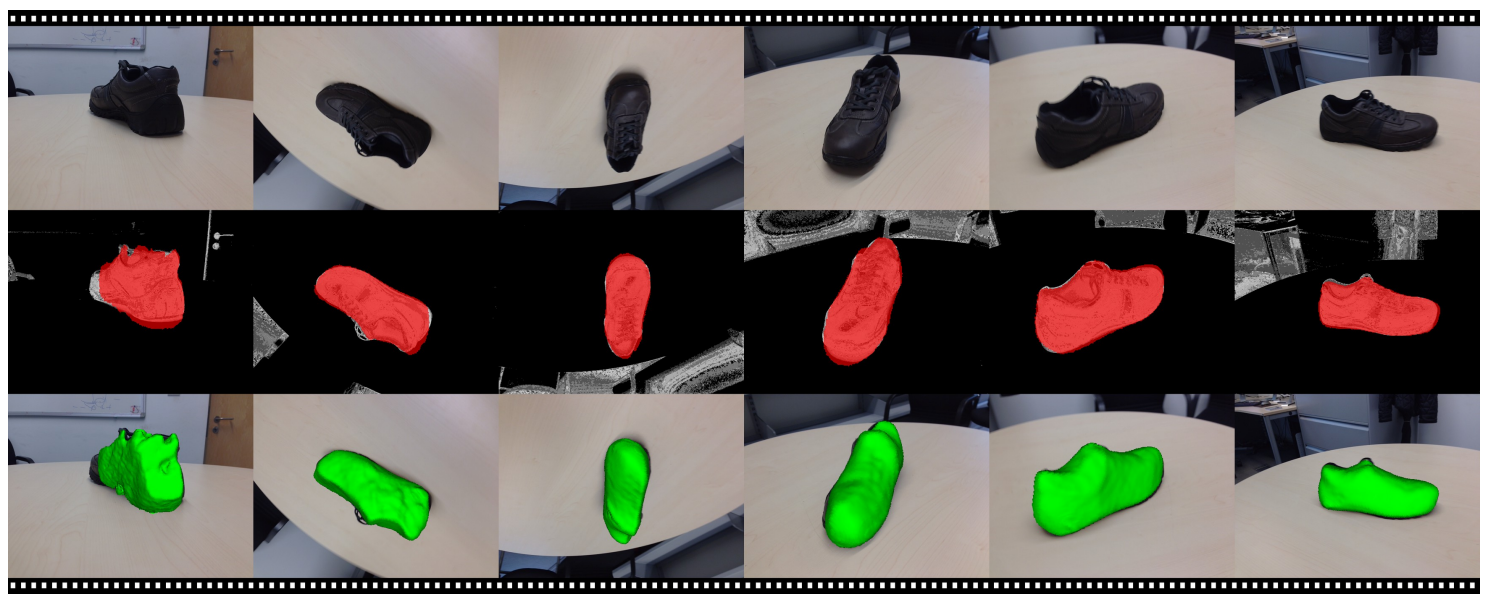

Fig. 13. Shoe tracking and reconstruction example. 


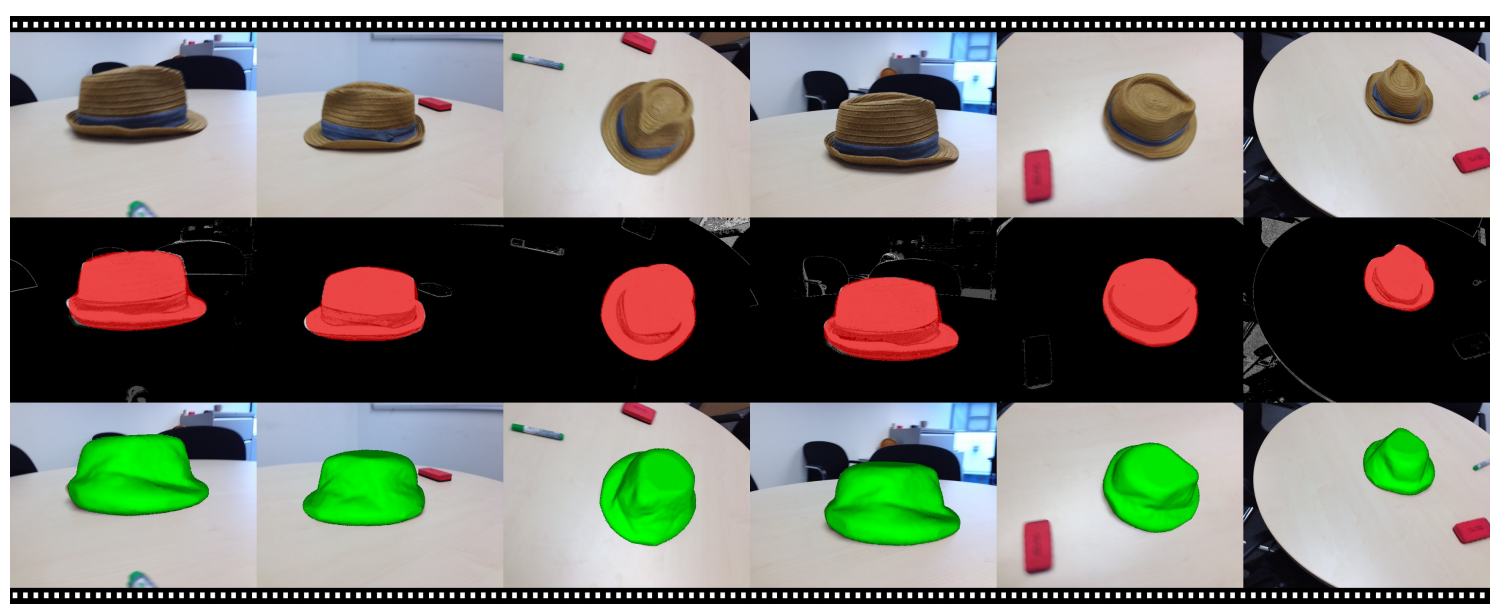

Fig. 14. Hat tracking and reconstruction example.

[13] V. A. Prisacariu, O. Kahler, D. Murray, and I. Reid. Simultaneous 3D tracking and reconstruction on a mobile phone. In ISMAR 2013, pages 89-98.

[14] V. A. Prisacariu and I. Reid. PWP3D: Real-Time Segmentation and Tracking of 3D Objects. IJCV 2012, pages 1-20.

[15] V. A. Prisacariu and I. Reid. Robust 3D hand tracking for human computer interaction. In $F G 2011$, pages 368-375.

[16] V. A. Prisacariu, A. Segal, and I. Reid. Simultaneous Monocular 2D Segmentation, 3D Pose Recovery and 3D Reconstruction. In ACCV 2012, pages 593-606.

[17] B. Rosenhahn, T. Brox, and J. Weickert. Three-Dimensional Shape Knowledge for Joint Image Segmentation and Pose Tracking. IJCV 2007, 73(3):243-262.

[18] H. Scharr. Optimal Operators in Digital Image Processing. PhD thesis, Heidelberg University, 2000.

[19] C. Schmaltz, B. Rosenhahn, T. Brox, J. Weickert, D. Cremers, L. Wietzke, and G. Sommer. Occlusion Modelling by Tracking Multiple Objects. In DAGM 2007, pages 173-183.

[20] D. Snow, P. Viola, and R. Zabih. Exact Voxel Occupancy with Graph Cuts. In CVPR 2000, pages 345-352.

[21] P. Tanskanen, K. Kolev, L. Meier, F. Camposeco, O. Saurer, and M. Pollefeys. Live Metric 3D Reconstruction on Mobile Phones. In ICCV 2013

[22] L. Vese and T. Chan. A Multiphase Level Set Framework for Image Segmentation Using the Mumford and Shah Model. IJCV 2002, 50(3):271-293.

[23] A. Yezzi and S. Soatto. Stereoscopic Segmentation. IJCV 2003, 53(1):31-43.

[24] S. You and U. Neumann. Fusion of vision and gyro tracking for robust augmented reality registration. In $V R$ 2001, pages 71-78.

[25] J. Yuan, E. Bae, and X.-C. Tai. A study on continuous max-flow and min-cut approaches. In CVPR 2010, pages 2217-2224.

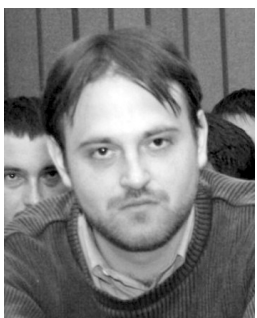

Victor Adrian Prisacariu graduated with 1st class honours in computer engineering in 2008 from the Gheorghe Asachi Tehnical University, lasi, Romania and completed his DPhil within the Department of Engineering Science, University of Oxford in 2012, where he was awarded an EPSRC doctoral training grant, a Balliol College Domus award and an EPSRC doctoral prize. He now works with within the same group as a post-doctoral research assistant. His research interests include semantic visual tracking, reconstruction and SLAM.

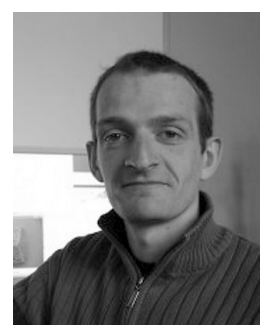

Olaf Kähler received the diploma degree in computer science from the FriedrichAlexander-University Erlangen-Nrnberg in 2004 and the doctorate degree from the Friedrich-Schiller-University Jena in 2009. From 2010 to 2011, he has been a research associate with the Machine Intelligence Lab at the University of Cambridge and since 2011 he is with the Active Vision Lab at the University of Oxford. His research interlem and semantic SLAM. ests include the structure-from-motion prob-

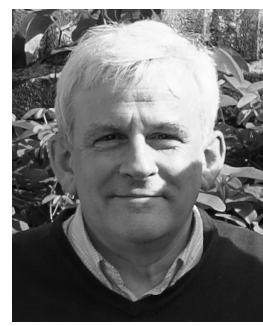

David Murray graduated with 1st class honours in physics in 1977 from the University of Oxford and completed his doctorate there in 1980 in low-energy nuclear physics. He was Research Fellow in physics at Caltech before moving to GEC's laboratories in London, where he developed research in structure from motion, motion segmentation and object recognition. He joined the University of Oxford in 1989 as a Fellow of St Anne's College and University Lecturer in the Department of Engineering Science, where he founded the Active Vision Laboratory. He was made a Professor of Engineering Science in 1997. His interests centre on active and ego-centric approaches to visual perception.

Ian D. Reid is a Professor of Computer Science and an ARC Australian Laureate Fellow at the University of Adelaide. Between 2000 and 2012 he was a Professor of Engineering Science at the University of Oxford. He received a BSc in Computer Science and Mathematics with first class honours from University of Western Australia in 1987 and was awarded a Rhodes Scholarship in 1988 to study at the University of Oxford, where he obtained a D.Phil. in 1992. Between then and 2000 when he was appointed to a Lecturership in Oxford, he held various Research Fellowship posts including an EPSRC Advanced Research Fellowship. His research interests include active vision, visual tracking, SLAM, human motion capture and intelligent visual surveillance, with an emphasis on real-time implementations whenever possible. He has published 150 papers, winning prizes at BMVC '05, '09, '10, and CVPR '08. He serves on the program committees of various national and international conferences, including as Program Chair for the Asian Conference on Computer Vision 2014. He is also on the editorial board of IEEE T-PAMI and Computer Vision and Image Understanding. 